\title{
Isolation of a Potent Naturally Occurring Hallucinogen, Salvinorin A, from Salvia divinorum and Investigation of Its Photo-degradation
}

Luann Wong $^{1}$, Sarah Stevens ${ }^{2}$, Matt Hengel ${ }^{2}$ and Takayuki Shibamoto ${ }^{2^{*}}$

${ }^{1}$ Department of Civil and Environmental Engineering, University of California, Davis, CA 95616, USA

${ }^{2}$ Department of Environmental Toxicology, University of California, Davis, CA 95616, USA

*Corresponding author: Shibamoto T, Department of Environmental Toxicology, University of California, Davis, CA 95616 , USA, Tel: +1530752 4523; E-mail: tshibamoto@ucdavis.edu

Received: November 20, 2017; Accepted: December 21, 2017; Published: January 12, 2018

Copyright: @ 2018 Wong, et al. This is an open-access article distributed under the terms of the Creative Commons Attribution License, which permits unrestricted use, distribution, and reproduction in any medium, provided the original author and source are credited.

\begin{abstract}
Salvinorin A, which is present in the natural plant Salvia divinorum, is a unique non-nitrogenous compound with hallucinogenic activity. When salvinorin A isolated from leaves of Salvia divinorum was irradiated with $300 \mathrm{~nm}$ UV light in ethyl acetate, it degraded from $100 \mu \mathrm{g} / \mathrm{mL}$ to $2.84 \pm 0.05 \mu \mathrm{g} / \mathrm{mL}$ in $30 \mathrm{~min}$. The calculated average rate constant $\mathrm{k}$ of this degradation was $0.12 / \mathrm{min}$ and the half-life was $5.7 \mathrm{~min}$. When authentic salvinorin $A$ was irradiated by UV light in an organic solution or an aqueous solution, it degraded over $90 \%$ within 40 min, whereas when it was irradiated by natural sunlight, it took $8 \mathrm{~h}$ to degrade $50 \%$ both in an organic and an aqueous solution. Three photodegradation products were tentatively identified by gas chromatography/mass spectrometry. Their structures were similar to that of salvinorin A, suggesting that they are also candidate non-nitrogenous hallucinogens.
\end{abstract}

Keywords: Salvia divinorum; Salvinorin A; Hallucinogens; Photodegradation

\section{Introduction}

Salvia divinorum is a rare plant species that grew in obscurity until its accidental discovery in 1962 [1]. Growth occurs through branching of dangling nodes and then through rooting at the nodes, creating the bush-like appearance of the plant, which has been known to grow up to a maximum of three meters in height [2,3]. In early 1980, salvinorin A was discovered in this plant as a psychoactive component $[4,5]$. Salvinorin A is a unique chemical as it is the only non-nitrogenous hallucinogen and psychoactive diterpene [6] because hallucinogens occurring in natural plants, such as mushrooms, are generally nitrogen-containing compounds [7]. Later, several salvinorin A related compounds, such as salvinorin B, salvidivins A, and salvinicin A were identified in Salvia divinorum; these are also unique to the plant $[8,9]$. These chemicals have been candidates for the psychoactive component of the plant because their structures are similar to that of salvinorin A. However, among these chemicals, only salvinorin A possesses significant hallucinogenic activity [5], suggesting that salvinorin A is the compound responsible for the hallucinogenic effect of Salvia divinorum [10]

Traditionally, salvia was used by the native Mazatec Indians from Oaxaca, Mexico, for divination, medico-religious ceremonies, and for its psychoactive effects [11]. In current times Salvia divinorum can be called salvia, magic mint, sally d, diviner's sage, or Purple Sticky, a particular brand [12]. Use of salvia is increasing as an alternative to marijuana $[13,14]$. Due to the dangers of smoking Salvia divinorum it has become illegal in several countries as well as in some states in the U.S. In order to establish regulations on the use of salvia to protect users, there is a pressing need to investigate various aspects of its nature, including its fate in the environment and its effects on humans after ingestion. Like many pharmaceutical compounds and illicit drugs, salvinorin A is unstable in the presence of light, but there are not many studies about salvinorin A and much is still unknown about it. Of particular import, there is no detailed information on its degradation pathway. In the present study, the behavior of salvinorin A under simulated and natural sunlight was investigated and tentative photo-degradation pathways of the products yielded was proposed.

\section{Materials and Methods}

\section{Chemicals and materials}

Salvinorin A (purity over 99\%) was purchased from Sigma-Aldrich (Saint Louis, MO, USA). It was also bought online from Cayman Chemical Company (Ann Arbor, MI, USA). HPLC grade acetone, dichloromethane, ethyl acetate, and methanol were purchased from Fisher Scientific (Fair Lawn, NJ, USA). Salvinorin A was also isolated from dried Salvia divinorum leaves, which were purchased from SalviaSupply.com (Road Town, Virgin Island, UK).

\section{Isolation of salvinorin A from Salvia divinorum}

Dried Salvia divinorum leaves ( $20 \mathrm{~g}$ ) were ground to a coarse power in a blender and then extracted twice with $1 \mathrm{~L}$ of acetone using a $2 \mathrm{~L}$ separatory funnel. After the acetone was removed by evaporation, the residual materials were transferred into a $200 \mathrm{~mL}$ separatory funnel containing $50 \mathrm{~mL}$ each of deionized water and ethyl acetate. The separatory funnel was shaken for $5 \mathrm{~min}$ and then the ethyl acetate layer was placed into a rotary evaporator to concentrate down to approximately $5 \mathrm{~mL}$ in volume. After the ethyl acetate solution was dried over minimum amount of anhydrous sodium sulfate. It was placed onto a $40 \mathrm{~cm} \times 4.5 \mathrm{~cm}$ i.d. glass column packed with silica gel and eluted with an acetone/dichloromethane (5/95) solution. Over twenty fractions of $10 \mathrm{~mL}$ each were collected and analyzed for the presence of salvinorin A using gas chromatography/mass spectrometry (GC/MS). Identification of salvinorin A was confirmed comparing the mass spectral fragmentation and GC retention time of authentic 
Citation: Wong L, Stevens S, Hengel M, Shibamoto T (2018) Isolation of a Potent Naturally Occurring Hallucinogen, Salvinorin A, from Salvia divinorum and Investigation of Its Photo-degradation. Nat Prod Chem Res 6: 305. doi:10.4172/2329-6836.1000305

Page 2 of 6

salvinorin A purchased from the commercial sources. Fractions found to contain salvinorin A were condensed and recrystallized using methanol to provide a $95 \%$ pure salvinorin $\mathrm{A}$.

\section{Photo-irradiation of salvinorin A from Salvia divinorum with a photo-reactor}

An ethyl acetate solution of salvinorin A $(100 \mu \mathrm{g} / \mathrm{mL})$ was placed into 8 quartz tubes $(5 \mathrm{~mL}$ each), which were subsequently irradiated with UV light $(\lambda=300 \mathrm{~nm})$ in a Rayonet RPR-100 photochemical reactor (Branford, CT, USA) equipped with 163000 RPR UV bulbs. A tube was removed at each of the following time intervals, 1, 3, 5, 10, 15, 20, 25, and $30 \mathrm{~min}$. Each reaction solution was analyzed for salvinorin A with the GC/FID.

Also, seven quartz tubes were filled with $4.2 \mathrm{~mL}$ each of aqueous solution $(20 \mu \mathrm{g} / \mathrm{mL})$ and irradiated with UV light. Tubes were removed after 5, 10, 20, 30, and $40 \mathrm{~min}$ of light exposure. An aqueous reaction solution from each tube $(4 \mathrm{~mL})$ was added to a Bond Elut C-18 solid phase extraction (SPE) cartridge (Varian, Lake Forest, CA, USA) and eluted with $3 \mathrm{~mL}$ ethyl acetate. The resulting effluent was analyzed for salvinorin A using the GC/FID. The cartridges were conditioned with 5 $\mathrm{mL}$ each of deionized water, methanol, and ethyl acetate in series prior to use.

Salvinorin A is not directly soluble in water. Therefore, $4.0 \mathrm{mg}$ of salvinorin A was originally dissolved in $10 \mathrm{~mL}$ methanol to get a 400 $\mu \mathrm{g} / \mathrm{mL}$ solution. To create a $20 \mu \mathrm{g} / \mathrm{mL}$ solution in deionized water, 2.5 $\mathrm{mL}$ of salvinorin A solution in methanol was diluted to $50 \mathrm{~mL}$ with deionized water.

\section{Recovery efficiency test on salvinorin A from solid phase extraction (SPE)}

Three concentrations $(80,100$, and $150 \mu \mathrm{g} / \mathrm{mL})$ of $50 / 50$-water/ acetonitrile solution of standard salvinorin $\mathrm{A}$ were prepared and each solution was placed on a SPE cartridge of the type used above. After the water/acetonitrile was eluted, the cartridge was eluted with $5 \mathrm{~mL}$ ethyl acetate to recover salvinorin A. The ethyl acetate eluates were analyzed for salvinorin A by gas chromatography.

\section{Photo-irradiation of salvinorin A with natural sunlight}

An ethyl acetate solution $(150 \mu \mathrm{g} / \mathrm{g})$ of salvinorin $\mathrm{A}(1 \mathrm{~mL})$ was placed in 24 quartz tubes. One test tube was wrapped with aluminum foil and used as a control. One tube was removed every $1 \mathrm{~h}$ and analyzed for degradation products. The experiment was carried out on October 8, 2014, on the roof of Meyer Hall at the University of California, Davis. The samples were exposed from 9 am to $5 \mathrm{pm}$. The weather was sunny and clear throughout the day.

Also, $1 \mathrm{~mL}$ each of a solution (water/acetonitrile $=50 / 50$ ) containing $100 \mu \mathrm{g} / \mathrm{g}$ salvinorin A was placed in 24 quartz test tubes. The control test tube was wrapped with foil. All tubes were exposed to natural sunlight and analyzed in triplicate. Test tubes were removed every hour. The experiment was carried out on February 24, 2015, on the roof of Meyer Hall at the University of California, Davis. The samples were exposed from 9:15 am to 5:15 pm. The weather was sunny and clear. After irradiation, the samples were prepared in ethyl acetate solution using the SPE described above for GC/MS analysis.

\section{Instrumental}

The irradiated samples were analyzed for salvinorin A by an Agilent 6890 series gas chromatograph coupled with a flame ionization detector (GC/FID). The GC was equipped with a $15.0 \mathrm{~m} \times 250 \mu \mathrm{m} \times$ $0.1 \mu \mathrm{m}$ DB-1 fused silica capillary column (Aligent, Folsom, CA, USA).

Helium carrier gas flow rate was $1.0 \mathrm{~mL} / \mathrm{min}$. The GC oven temperature was held at $150^{\circ} \mathrm{C}$ for $1 \mathrm{~min}$ and then programmed to rise to $250^{\circ} \mathrm{C}$ at $30^{\circ} \mathrm{C} / \mathrm{min}$. The injector and detector temperatures were $300^{\circ} \mathrm{C}$ and $280^{\circ} \mathrm{C}$, respectively.

All standards and irradiated samples were also analyzed by an Agilent Technologies $6890 \mathrm{~N}$ Gas Chromatograph interfaced to an Agilent Technologies 5973 Network Mass Selective Detector (GC/ MSD). The column used in the GC system was a $30 \mathrm{~m} \times 250 \mu \mathrm{m} \times 0.25$ $\mu \mathrm{m}$ DB-1 fused silica capillary column (Aligent, Folsom, CA, USA). Helium carrier gas flow rate was $1.0 \mathrm{~mL} / \mathrm{min}$. The inlet and the GC/MSD interface were set to $250^{\circ} \mathrm{C}$ and $280^{\circ} \mathrm{C}$, respectively. The initial oven temperature was $80^{\circ} \mathrm{C}$ and was held for $2 \mathrm{~min}$, then programmed to rise to $280^{\circ} \mathrm{C}$ at $20^{\circ} \mathrm{C} / \mathrm{min}$. The final temperature was held for $8 \mathrm{~min}$. The electron energy of the MS was $70 \mathrm{eV}$. The quadrupole mass spectrometer was set to scan from 50 to $550 \mathrm{~m} / \mathrm{z}$. The temperatures of the ionization source and the quadrupole were $230^{\circ} \mathrm{C}$ and $150^{\circ} \mathrm{C}$, respectively.

Tentative identification of photo-degradation products was performed using our knowledge for elucidation of structures based on MS fragmentation. Also, the NIST02 mass spectral library (National Institute of Standards and Technology, Gaithersburg, MD, USA) and salvinorin A related compounds reported previously [15] were referred to for further confirmation of our identifications.

\section{Results and Discussion}

As mentioned above, preparation of an aqueous salvinorin A is not easy because of its insolubility in water. However, salvinorin A was readily dissolved in a solution of water/acetonitrile (50/50). The preliminary experiments on photo-irradiation of salvinorin $\mathrm{A}$ in aqueous and water/acetonitrile solutions showed no significant difference. Therefore, a water/acetonitrile solution was used for further photo-degradation experiments.

The limit of detection (LOD) and the limit of quantitation for salvinorin A in an ethyl acetate solution determined according to a previously reported method [16] were $7.02 \mu \mathrm{g} / \mathrm{mL}$ and $23.4 \mu \mathrm{g} / \mathrm{mL}$, respectively. These values are relatively high, but represent a very conservative estimate, since the instrument could actually produce a measurable peak at a concentration of $0.50 \mu \mathrm{g} / \mathrm{mL}$ in the present study.

The recovery efficiencies of SPE used were $79.82 \pm 0.80 \%$ for 80 $\mu \mathrm{g} / \mathrm{mL}, 99.78 \pm 1.94 \%$ for $100 \mu \mathrm{g} / \mathrm{mL}$, and $94.35 \pm 3.73 \%$ for $150 \mu \mathrm{g} / \mathrm{mL}$ from a water/acetonitrile solution.

Figure 1 shows the photo-degradation of salvinorin A isolated from the leaves of Salvia divinorum in ethyl acetate irradiated with $300 \mathrm{~nm}$ $\mathrm{UV}$ light for $30 \mathrm{~min}$. Values are mean $\pm \mathrm{SD}(\mathrm{n}=3)$. SD ranged from \pm 5.32 ( 3 min irradiation) to \pm 0.03 ( 25 min irradiation) but the values less than \pm 2.30 do not appear in Figure 1. The degradation occurred rather quickly. Salvinorin A $(100 \mu \mathrm{g} / \mathrm{mL})$ was degraded down to $2.84 \pm$ $0.05 \mu \mathrm{g} / \mathrm{mL}$ after $30 \mathrm{~min}$. The calculated average rate constant $\mathrm{k}$ of this degradation was $0.12 / \mathrm{min}$ and the half-life was $5.7 \mathrm{~min}$. The degradation rate constant and half-life were calculated using the following first-order reaction equations [17]: 
Citation: Wong L, Stevens S, Hengel M, Shibamoto T (2018) Isolation of a Potent Naturally Occurring Hallucinogen, Salvinorin A, from Salvia divinorum and Investigation of Its Photo-degradation. Nat Prod Chem Res 6: 305. doi:10.4172/2329-6836.1000305

Page 3 of 6

$\operatorname{In}[\text { Salvinorin } \mathrm{A}]_{\mathrm{t}} /[\text { Salvinorin } \mathrm{A}]_{0}=-\mathrm{kt}$ and $\mathrm{t}_{1 / 2}=\operatorname{In} 2 / \mathrm{k}$

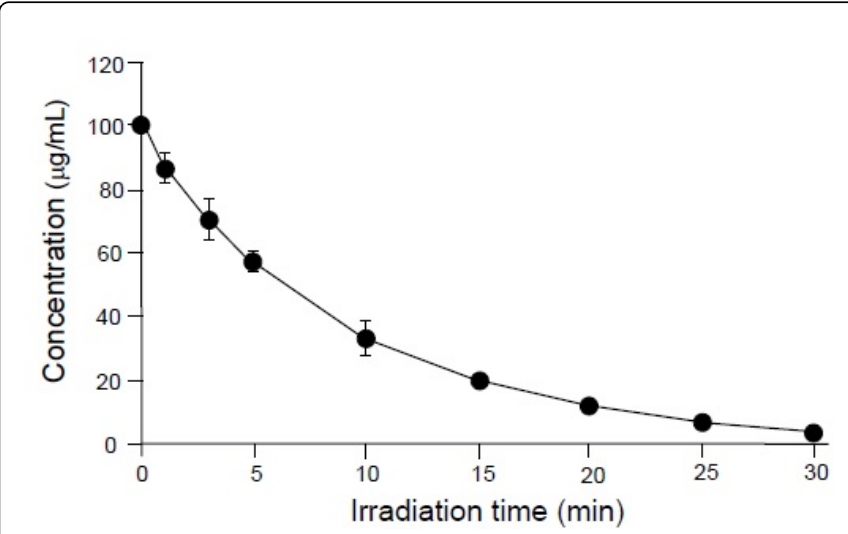

Figure 1: Results of photo-degradation of salvinorin A isolated from the leaves of Salvia divinorum in ethyl acetate irradiated with 300 $\mathrm{nm}$ UV light for $30 \mathrm{~min}$.

Where [Salvinorin $\mathrm{A}]_{t}$ represents the concentration of salvinorin $\mathrm{A}$ at time $\mathrm{t}$, [Salvinorin $\mathrm{A}]_{0}$ represents the concentration of salvinorin $\mathrm{A}$ at time $0, \mathrm{k}$ represents the rate constant, and $t_{1 / 2}$ is the half-life. The rate constants were determined by plotting the graphs of $\operatorname{In}[$ Salvinorin $\mathrm{A}]_{\mathrm{t}} /[\text { Salvinorin } \mathrm{A}]_{0}$ against time.

Figure 2 shows the photo-degradation of salvinorin A isolated from leaves of Salvia divinorum in water irradiated with $300 \mathrm{~nm}$ UV light for $40 \mathrm{~min}$. Salvinorin A $(20 \mu \mathrm{g} / \mathrm{mL})$ was degraded down to $3.15 \pm 0.01$ $\mu \mathrm{g} / \mathrm{mL}$ after $40 \mathrm{~min}$. The calculated average rate constant of this degradation was $0.044 / \mathrm{min}$ and the half-life time was $16 \mathrm{~min}$. When salvinorin A isolated from Salvia divinorum leaves was irradiated with $300 \mathrm{~nm}$ UV light in an ethyl acetate or water solution, it degraded almost three times slower in water than in ethyl acetate.

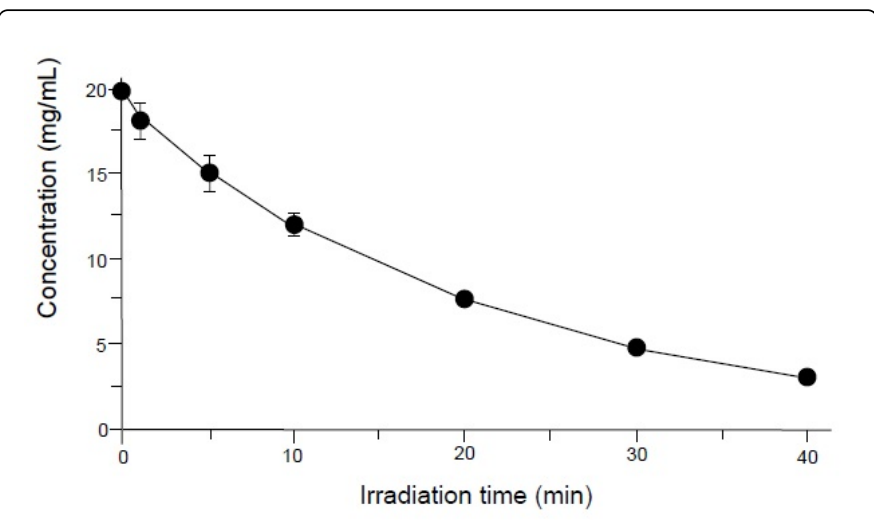

Figure 2: Results of photo-degradation of salvinorin A isolated from leaves of Salvia divinorum in water irradiated with $300 \mathrm{~nm}$ UV light for $40 \mathrm{~min}$.

Figure 3 shows the photo-degradation of authentic salvinorin $\mathrm{A}$ in ethyl acetate and water/acetonitrile (50/50) solutions irradiated with $300 \mathrm{~nm}$ UV light for $40 \mathrm{~min}$. Values are mean \pm SD $(\mathrm{n}=3)$. In the case of the ethyl acetate solution, SD ranged from \pm 4.95 (10 $\mathrm{min}$ irradiation) to \pm 0.05 (20 min irradiation); values less than \pm 2.60 do not appear in Figure 3. Authentic salvinorin A degraded from 100 $\mu \mathrm{g} / \mathrm{mL}$ to $8.33 \mu \mathrm{g} / \mathrm{mL}$ after $30 \mathrm{~min} \mathrm{UV}$ irradiation. The calculated average rate constant of this degradation was $0.8 / \mathrm{min}$ and the half-life time was $8.7 \mathrm{~min}$.

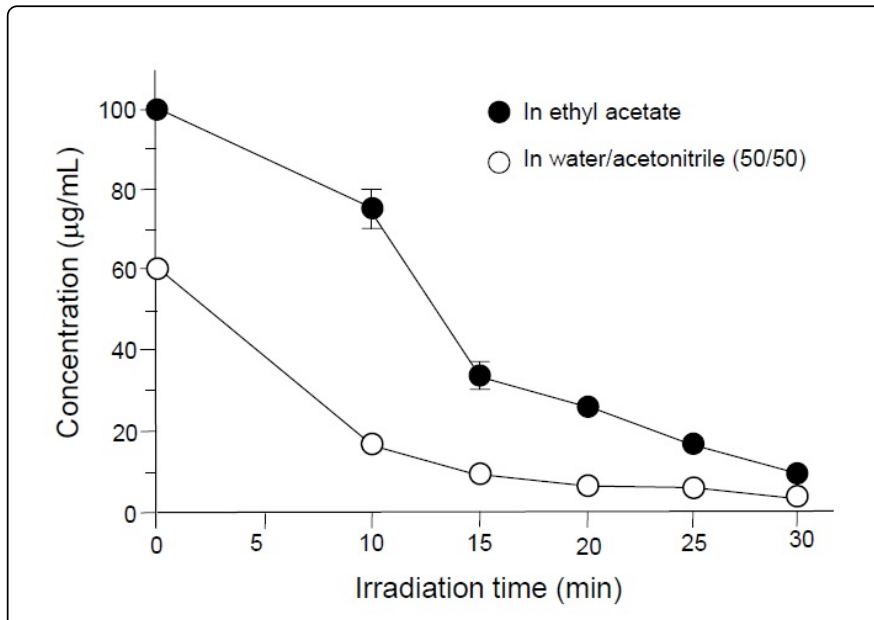

Figure 3: Results of photo-degradation of authentic salvinorin A in ethyl acetate and water/acetonitrile (50/50) solutions irradiated with $300 \mathrm{~nm}$ UV light for $40 \mathrm{~min}$.

In the case of the water/acetonitrile (50/50) solution, SD ranged from \pm 1.09 ( $10 \mathrm{~min}$ irradiation) to \pm 0.01 ( $25 \mathrm{~min}$ irradiation $)$; values less than \pm 2.60 do not appear in Figure 3. Authentic salvinorin A degraded from $60 \mu \mathrm{g} / \mathrm{mL}$ to $2.86 \mu \mathrm{g} / \mathrm{L}$ after $30 \mathrm{~min}$ UV irradiation. The calculated average rate constant of this degradation was $0.09 / \mathrm{min}$ and the half-life time was $7.7 \mathrm{~min}$.

Figure 4 shows the photo-degradation of authentic salvinorin $\mathrm{A}$ in ethyl acetate and water/acetonitrile (50/50) solutions irradiated with natural sunlight for $30 \mathrm{~min}$. Values are mean $\pm \mathrm{SD}(\mathrm{n}=3)$. In the case of an ethyl acetate solution, SD ranged from \pm 8.16 ( $5 \mathrm{~h}$ irradiation) to \pm 2.51 ( $2 \mathrm{~h}$ irradiation); values less than \pm 2.91 do not appear in Figure 4 . Authentic salvinorin A degraded from $150 \mu \mathrm{g} / \mathrm{mL}$ to $71.89 \mu \mathrm{g} / \mathrm{mL}$ after 40 min sunlight irradiation. The calculated average rate constant of this degradation was $0.08 / \mathrm{h}$ and the half-life time was $8.84 \mathrm{~h}$.

In the case of the water/acetonitrile (50/50) solution, SD ranged from \pm 9.13 ( $5 \mathrm{~min}$ irradiation) to \pm 2.54 (40 min irradiation); values less than \pm 2.91 do not appear in Figure 4. Authentic salvinorin A degraded from $100 \mu \mathrm{g} / \mathrm{mL}$ to $40.03 \mu \mathrm{g} / \mathrm{mL}$ after $40 \mathrm{~min}$ sunlight irradiation. The calculated average rate constant of this degradation was $0.10 / \mathrm{h}$ and the half-life time was $6.82 \mathrm{~h}$.

All figures exhibited similar patterns of first-order degradation curves. When salvinorin A from Salvia divinorum leaves was irradiated with $300 \mathrm{~nm}$ UV light in ethyl acetate or water, degradation occurred much faster in ethyl acetate than in water. This may be due to its lessened solubility in water because it degraded in water/acetonitrile solution almost as fast as in ethyl acetate. Also, Salvia divinorum leaves contain some water soluble chemicals which inhibit the photodegradation of salvinorin $\mathrm{A}$. The results indicate that salvinorin $\mathrm{A}$ degrades readily by UV light, suggesting that it is important to prevent exposure to UV light for determination of the levels of salvinorin A in natural plants after collection. 
Citation: Wong L, Stevens S, Hengel M, Shibamoto T (2018) Isolation of a Potent Naturally Occurring Hallucinogen, Salvinorin A, from Salvia divinorum and Investigation of Its Photo-degradation. Nat Prod Chem Res 6: 305. doi:10.4172/2329-6836.1000305

Page 4 of 6

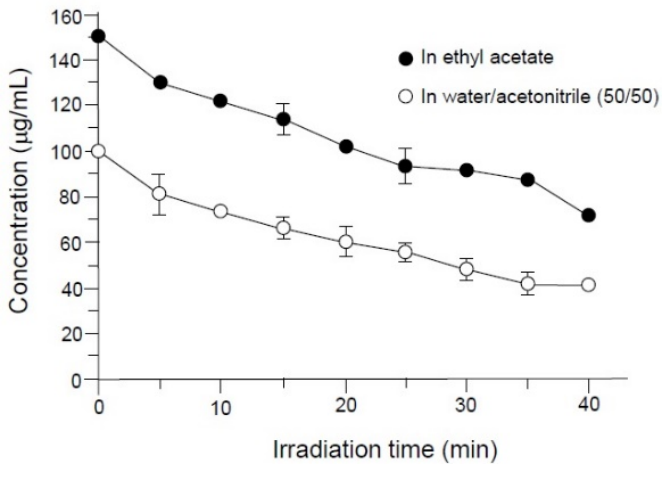

Figure 4: Results of photo-degradation of authentic salvinorin A in ethyl acetate and water/acetonitrile (50/50) solutions irradiated with natural sunlight for $30 \mathrm{~min}$.
The degradation rates calculated are at a rather accelerated rate based on high UV exposure. A study of the metabolism of salvinorin A in rat plasma found a degradation rate of $0.11 / \mathrm{h}$ and a half-life of 6.30 $\mathrm{h}$ at $25^{\circ} \mathrm{C}$ [18]. This study also found that increasing the temperature increased the rate of degradation with less than two percent of the original salvinorin A present in the sample after incubation at $37^{\circ} \mathrm{C}$, or body temperature, for $24 \mathrm{~h}$. However, the degradation rate of salvinorin A by a living organism is comparable to that of UV irradiation. The quick breakdown of salvinorin A in living organisms may account for the short duration of its hallucinogenic effects.

Figure 5 shows a typical gas chromatogram of a sunlight irradiated (20 min) sample in ethyl acetate. Refer to Table 1 for details of the degradation products tentatively identified. Gas chromatography has been used widely for analysis of plant extracts [19].

\begin{tabular}{|c|c|c|c|}
\hline Compound & Systematic name & $\begin{array}{l}\text { GC retention } \\
\text { time (min) }\end{array}$ & MS fragment, $\mathrm{m} / \mathbf{z}(\%)$ \\
\hline Salvinorin A & $\begin{array}{l}\text { methyl (2S,4aR,6aR,7R,9S,10aS,10bR)-9- } \\
\text { (acetyloxy)-2-(furan-3-yl)-6a,10b- } \\
\text { dimethyl-4,10-dioxo-dodecahydro-1H- } \\
\text { naphtho[2,1-c]pyran-7-carboxylate }\end{array}$ & 18.26 & $\begin{array}{l}432(\mathrm{M}+, 11.0), 404(8.5), 359(8.0), 318(6.4), 273(23.8), 220(12.1), 166 \\
(15.6), 121(19.7), 94(100), 43(41.9)\end{array}$ \\
\hline Compound I & $\begin{array}{l}\text { methyl }(4 S, 4 a R, 5 S, 6 R, 8 a R)-5-[2-(3- \\
\text { furyl)ethenyl]-4- } \\
\text { hydroxy-6(hydroxymethyl)-5,8a- } \\
\text { dimethyl-3,4,4a,5,6,7,8,8a-octahydro-1- } \\
\text { naphthalenecarboxylate }\end{array}$ & 14.04 & $\begin{array}{l}360(\mathrm{M}+, 33.1), 342(6.4), 328(8.3), 301(85.7), 283(18.6), 245(37.6), 121 \\
(100), 107(56.6), 95(80.8), 81(58.0), 55(31.6)\end{array}$ \\
\hline Compound II & $\begin{array}{l}\text { methyl }(6 a R, 7 R, 10 b R)-2-(f u r a n-3-y l)-6 a, \\
\text { 10bdimethyl-4,10 } \quad \text {-dioxo-1,4,4a,5,6,6a, } \\
\text { 7,10,10a,10b-decahydro-2Hbenzo[f] } \\
\text { isochromene-7-carboxylate }\end{array}$ & 14.80 & $\begin{array}{l}372(\mathrm{M}+, 64.4), 340(9.4), 312(100), 297(39.9), 253(16.1), 173(39.7), 159 \\
(28.6), 145(64.2), 131(35.2), 105(28.2), 91(47.6), 55(19.2)\end{array}$ \\
\hline Compound III & $\begin{array}{l}\text { methyl (2R, E)-2-((4aR,6R)-5-formyl-3- } \\
\text { (furan3-yl)-4a,6-dimethyl r } r-1- \\
\text { oxooctahydro-1Hisochromen-6-yl)-4-(prop-1- } \\
\text { en-2-yloxy) but-3enoate }\end{array}$ & 17.20 & $\begin{array}{l}432(\mathrm{M}+, 15.1), 404(25.8), 318(12.6), 273(79.6) \\
2.6(33.4), 146(28.6), 121(47.5), 107(22.5) \\
94 \text { (100), } 81 \text { (13.2), } 55(26)\end{array}$ \\
\hline
\end{tabular}

Table 1: Photo-degradation products and salvinorin A identified in an ethyl acetate solution of authentic salvinorin A irradiated with $300 \mathrm{~nm}$ UV light. 
Citation: Wong L, Stevens S, Hengel M, Shibamoto T (2018) Isolation of a Potent Naturally Occurring Hallucinogen, Salvinorin A, from Salvia divinorum and Investigation of Its Photo-degradation. Nat Prod Chem Res 6: 305. doi:10.4172/2329-6836.1000305

Page 5 of 6

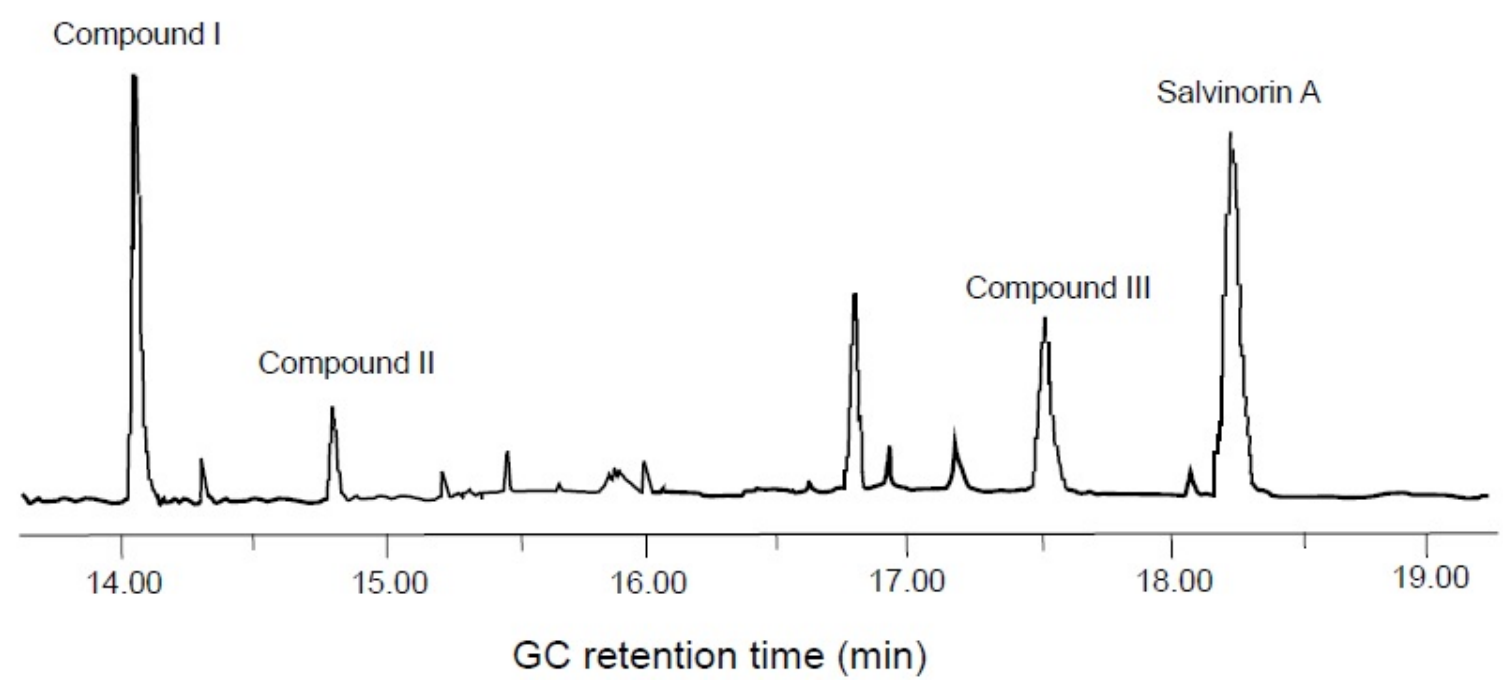

Figure 5: A typical gas chromatogram of sunlight irradiated $(20 \mathrm{~min})$ sample in ethyl acetate.

Figure 6 presents proposed formation pathways of these compounds. Identification of salvinorin A in this sunlight irradiated solution was confirmed using an authentic chemical purchased from commercial sources. Compound II was proposed to form from salvinorin A via a Norrish type I photo-reaction cleaving a bond between carbon numbers 1 and 2 . Intermediate $A$ was formed via hemolytic cleavage between carbon number 2 and acetate oxygen and subsequently Compound II was formed upon losing a hydrogen radical. Compound I may form through intermediate $B$ from Compound II as shown in Figure 6. However, formation of this compound may come from other products. A previous study reported the presence of a similar compound, which has a saturated bond between carbon numbers 11 and 12 [15]. They proposed the structure of this compound using the ${ }^{1} \mathrm{H}$ NMR spectrum.

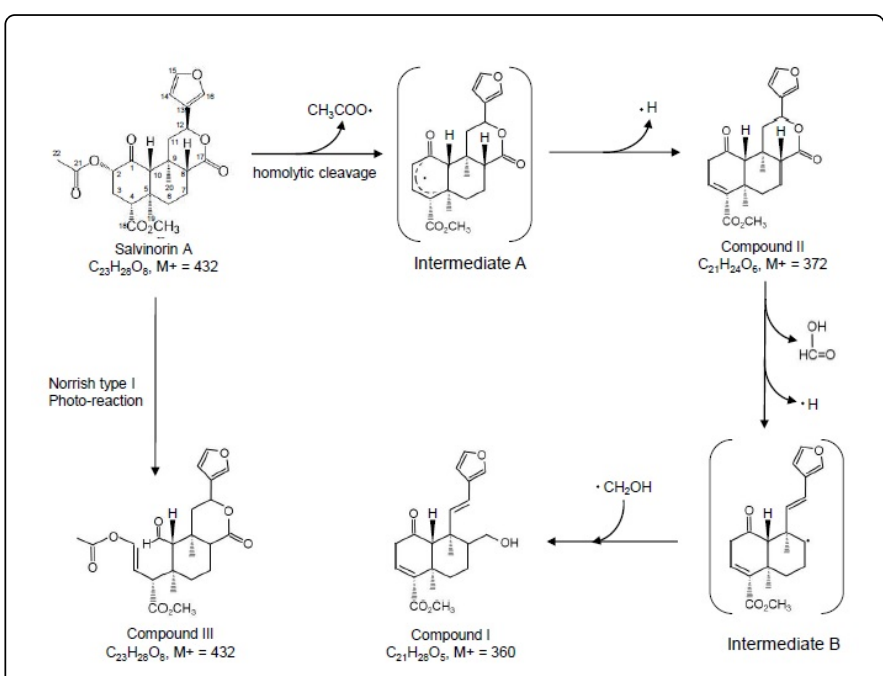

Figure 6: Proposed formation pathways of the compounds tentatively identified in an ethyl acetate solution irradiated by sunlight for $20 \mathrm{~min}$.

\section{Conclusion}

Naturally occurring hallucinogens containing no nitrogen atom, such as salvinorin A, are unique. Photo-degradation of salvinorin A occurs in relatively short time, suggesting that it is necessary to store in appropriate light-free conditions. In addition, salvinorin A produces various photo-degradation compounds, which may possess hallucinogenic effects. In the present study, salvinorin A isolated from a natural plant, Salvia divinorum, behaved the same as the authentic chemical. Detailed study of the hallucinogenic activity of these photodegradation products is in order.

\section{References}

1. Wasson RG (1962) A new Mexican psychotropic drug from the mint family. Bot Mus Leaflets Harvard Univ 20: 77-84.

2. Reisfeld AS (1993) The Botany of Salvia divinorum (Labiatae). SIDA 15: 349-366.

3. Valdés LJIII, Hatfield GM, Koreeda M, Paul AG (1987) Studies of Salvia divinorum (Lamiaceae), an hallucinogenic mint from the Sierra Mazateca in Oaxaca, central Mexico. Econ Bot 41: 283-291.

4. Ortega A, Blount JF, Manchand PS (1982) Salvinorin, a new transneoclerodane diterpene from Salvia divinorum (Labiatae). J Chem Soc Perkin Trans 1: 2505-2508.

5. Valdés LJIII, Butler WM, Hatfield GM, Paul AG, Koreeda M (1984) Divinorin A, a psychotropic terpenoid, and divinorin B from the hallucinogenic Mexican mint, Salvia divinorum. Org Chem 49: 4716-4720.

6. Vortherms TA, Roth BL (2006) Salvinorin A: From natural product to human therapeutics. Mol Interventions 6: 257-265.

7. Nagy J, Veress T (2016) HPLC analysis of hallucinogenic mushroom alkaloids (Psilocin and psilocybin) applying hydrophilic interaction chromatography (HILIC). J Foren Res 7: 356-372.

8. Harding WW, Tidgewell K, Schmidt M, Shah K, Dersch CM, et al. (2005) Salvinicins A and B, ne neoclerodane diterpenes from Salvia divinorum. Org Let 7: 3017-3020.

9. Shirota O, Nagamatsu K, Sekita S (2007) Neo-clerodane diterpenes from the hallucinogenic sage Salvia divinorum. J Nat Prod 69: 1782-1786. 
Citation: Wong L, Stevens S, Hengel M, Shibamoto T (2018) Isolation of a Potent Naturally Occurring Hallucinogen, Salvinorin A, from Salvia divinorum and Investigation of Its Photo-degradation. Nat Prod Chem Res 6: 305. doi:10.4172/2329-6836.1000305

Page 6 of 6

10. Roth BL, Baner K, Westkaemper R, Siebert D, Rice KC, et al. (2002) Salvinorin A: A potent naturally occurring nonnitrogenous $\kappa$ opioid selective agonist. PNAS 99: 11934-11939.

11. Siebert DJ (2004) Localization of Salvinorin A and related compounds in glandular trichomes of the psychoactive sage, Salvia divinorum. Ann Bot 93: 763-771.

12. Halpern JH, Pope JG (2001) Hallucinogens on the internet: A vast new source of underground drug information. Am J Psychiatry 158: 481-483.

13. González D, Riba J, Bouso JC, Gómez-Jarabo G, Barbanoj MJ (2006) Pattern of use and subjective effects of Salvia divinorum among recreational users. Drug Alcohol Depend 85: 157-162.

14. Lange JE, Reed MB, Ketchie Croff JM, Clapp JD (2008) College student use of Salvia divinorum. Drug Alcohol Depend 94: 263-266.

15. Bigham AK, Munro TA, Rizzacasa MA, Robins-Browne RM (2003) Divinatorins A-C, new neoclerodane diterpenoids from the controlled sage Salvia divinorum. J Nat Prod 66: 1242-1244.
16. Saadati N, Abdullah MP, Zakaria Z, Sany SB, Rezayi TM, et al. (2013) Limit of detection and limit of quantification development procedures for organochlorine pesticides analysis in water and sediment matrices. Chem Cent J 7: 63-71.

17. Espino MPB (2008) Photolytical Degradation Products of Pentachlorophenol in Aqueous Solution and Organic Solvents. Philippine J Sci 137: 179-186.

18. Tsujikawa K, Kuwayama K, Miyaguchi H, Knanmori T, Iwata YT, et al. (2008) Determination of salvinorin A and salvinorin B in Salvia divinorum-related products circulated in Japan. Forensic Sci Int 180: 105-109.

19. Al-Ghamd AK, Elkholy TA, Abuhelal S, Alabbadi H, Qahwaji K, et al. (2017) Study of Jojoba (Simmondsia chinensis) oil by gas chromatography. Nat Prod Chem Res 5: 5-9. 